\title{
Optical Properties and Application of Uranium-based Thin Films for the Extreme Ultraviolet and Soft X-ray Region
}

\author{
Richard L. Sandberg \\ rudyskaboy@yahoo.com \\ David D. Allred \\ allred@byu.edu \\ Shannon Lunt \\ Marie K. Urry \\ Follow this and additional works at: https://scholarsarchive.byu.edu/facpub \\ D Steven Turley \\ Part of the Astrophysics and Astronomy Commons, and the Physics Commons
}

\section{Original Publication Citation}

Richard L. Sandberg, David D. Allred, Shannon Lunt, Marie K. Urry, R. Steven Turley, "Optical Properties and Application of Uranium-based Thin Films for the Extreme Ultraviolet and Soft Xray Region," in Optical Constants of Materials for UV to X-Ray Wavelengths, edited by Regina Soufli, and John F. Seely, Proceedings of SPIE, Vol. 5538, (SPIE, Bellingham, WA, 24) 17-118. [http://spiedigitallibrary.org/proceedings/resource/2/psisdg/5538/1/ 17_1?isAuthorized=no][http://dx.doi.org/1.1117/12.56717].

\section{BYU ScholarsArchive Citation}

Sandberg, Richard L.; Allred, David D.; Lunt, Shannon; Urry, Marie K.; and Turley, R. Steven, "Optical Properties and Application of Uranium-based Thin Films for the Extreme Ultraviolet and Soft X-ray Region" (2004). Faculty Publications. 1041.

https://scholarsarchive.byu.edu/facpub/1041

This Peer-Reviewed Article is brought to you for free and open access by BYU ScholarsArchive. It has been accepted for inclusion in Faculty Publications by an authorized administrator of BYU ScholarsArchive. For more information, please contact ellen_amatangelo@byu.edu. 


\title{
Optical Properties and Application of Uranium-based Thin Films for the Extreme Ultraviolet and Soft $X$-ray Region
}

\author{
Richard L. Sandberg, David D. Allred*, Shannon Lunt, Marie K. Urry, R. Steven Turley \\ Department of Physics and Astronomy, Brigham Young University, \\ N-283 ESC, Provo, UT USA 84602
}

\begin{abstract}
Uranium oxide and uranium nitride thin films reflect significantly more than all previously known/standard reflectors (e.g., nickel, gold, and iridium) for most of the $4-10 \mathrm{~nm}$ range at low angles of incidence. This work includes measurements of the EUV/soft x-ray $(2-20 \mathrm{~nm})$ reflectance of uranium-based thin films ( $20 \mathrm{~nm}$ thick) and extraction of their optical constants $(d$ and $\beta)$. We report the reflectances at 5,10 , and 15 degrees grazing incidence of air-oxidized sputtered uranium, reactively sputtered $\left(\mathrm{O}_{2}\right)$ uranium oxide, and reactively sputtered $\left(\mathrm{N}_{2}\right)$ uranium nitride thin films measured at Beamline 6.3.2 at the Advanced Light Source (ALS) at Lawrence Berkeley National Laboratory (LBNL). Additionally, we report optical constants of reactively sputtered uranium oxide at nine wavelengths from 4.6 to $17.5 \mathrm{~nm}$ derived from ALS angle-scan reflectance measurements. We also report optical constants of uranium nitride at 13 and $14 \mathrm{~nm}$. We compare the reflectance of these uraniumcompound thin films to gold, nickel (and nickel oxide), and iridium thin films from 2.5 to $11.6 \mathrm{~nm}$. These metal thin films were chosen for comparison due to their wide use in EUV/soft x-ray applications as low-angle, thinfilm reflectors. The uranium compounds can exhibit some surface oxidation in ambient air. There are important discrepancies between $\mathrm{UO}_{2}$ 's and UN's actual thin-film reflectance with those predicted from tabulated optical constants of the elemental constituents of the compounds. These differences are also demonstrated in the optical constants we report. Uranium-based optics applications have important advantages for zone plates, thin-film reflectors, and filters.
\end{abstract}

Keywords: soft x-ray, EUV, low-angle reflector, nickel, uranium oxide, uranium nitride, synchrotron, EUV/soft $\mathrm{X}$-ray astronomy, optical constants

\section{INTRODUCTION}

We report our group's investigation of uranium compound thin films to increase the low-angle reflectance currently available for thin-film single-layer reflectors at 40-250 eV (5-9 nm) ${ }^{* 1,2,3,4,5}$ Additionally, we report on the methods used and results of a study to measure the optical constants $(d$ and $\beta)$ for reactively sputtered uranium oxide and reactively sputtered uranium nitride. Here we provide recent reflectance measurements from $100-460 \mathrm{eV}$ (2.7 to $11.6 \mathrm{~nm}$ ) comparing uranium [as naturally oxidized uranium (UO2), and uranium nitride (UN)], nickel (and nickel oxide), iridium, and gold thin-film reflectors. Nickel, iridium, and gold were chosen for the reflectance comparison because of their wide use as a thin-film reflective coating in optical systems for the EUV and soft x-ray range. ${ }^{6}$ The samples' reflectances were measured at the Advanced Light Source's Beamline 6.3.2 at the Lawrence Berkley National Laboratory. We find that the low-angle reflectance of uranium oxide and uranium nitride surfaces exceeds that of traditional coatings over a large wavelength range. We additionally report on the stability of these uranium compounds.

Uranium has a high predicted reflectance due to its high density and large number of electrons. However, uranium is chemically active and will quickly oxidize in ambient air. Uranium nitride has the highest uranium atom density of known compounds according to data in Cordfunke. ${ }^{7}$ Additionally, Black et al ${ }^{8}$ stated that uranium nitride is resistant to bulk oxidation. In contrast, Urry showed that uranium nitride thin films sputtered at room temperature exhibit some surface oxidation. ${ }^{2}$

*allred@byu.edu; phone 1801 422-3489; fax 1801 422-0553; http://xuv.byu.edu 
In March of 2000 the IMAGE (Imager for Magnetopause-to-Aurora Global Exploration) Satellite was launched carrying three multilayer uranium-based mirrors made by the EUV optics group at Brigham Young University. These mirrors have been used for imaging the magnetosphere of the earth. ${ }^{*}$ Other potential application for the uranium as a thin-film coating in this wavelength region include astronomical applications, medical imaging, and zone plate lenses. ${ }^{9}$

\section{EXPERIMENTAL}

All of the samples except the iridium sample were prepared here at BYU. The uranium-containing samples were deposited by magnetron sputtering: UN via RF sputtering in one system and the two kinds of uranium oxide (air oxidized uranium and reactive sputtered $\mathrm{UO}_{\mathrm{x}}$ ) via DC sputtering in another. ${ }^{2,5}$ The nickel and gold were deposited by thermal evaporation in a third system. All the BYU systems were cryopumped.

The iridium samples were sputtered at the Goddard Space Flight Center on glass slides. ${ }^{10}$ The other materials were deposited on a variety of substrates including the following: pieces of standard polished, silicon-test wafers (100 orientation), fused quartz slides, and carbon coated TEM (transmission electron microscope) grids. All samples were deposited at room temperature. The deposited films ranged from 10 to $50 \mathrm{~nm}$ in thickness. The surface roughness (RMS) of several silicon wafers was measured via atomic force microscopy (AFM) to be $\sim 0.2$ $\mathrm{nm}$ over a 100 by $100 \mathrm{~nm}$ area.*

\subsection{Uranium compounds deposited through sputtering}

All the uranium-based samples were sputtered in one of two stainless steel chambers from a uranium target (Manufacturing Sciences, Oak Ridge, TN) in independently controlled flowing argon, and if needed, reactive gases with a pressure range of 1 to $15 \times 10^{-3}$ torr. The uranium sputter targets used were of depleted uranium metal (less than $0.2 \%$ U-235). We calculated the decay per second of depleted uranium as about 0.238 alphas, twice as many betas and miscellaneous $\mathrm{L} x$ rays for each $\mathrm{cm}^{2}$ of a $10 \mathrm{~nm}$ film at bulk density. The users' solid angle of detection of these will be considerably less than $2 p$ steradians. (Half go down into the substrate and are absorbed.) So counts from the mirror will be small compared to background for soft x-ray applications.*

Two processes were used for depositing uranium oxide as described in previous works by the BYU EUV group. Both processes involved sputtering in a stainless steel vacuum chamber named Davy. One process was to sputter a uranium thin film and let it oxide in air. A $20 \mathrm{~nm}$ film is thoroughly oxidized in a day (TEM and x-ray photoelectron spectroscopy, or XPS, show oxidized uranium to be mostly $\left.\mathrm{UO}_{2}\right) .{ }^{11}$ Lunt reactively sputtered uranium in an oxygen partial pressure of $3 \times 10^{-4}$ torr. $^{5}$ More details are found in Lunt ${ }^{5}$ and Oliphant. ${ }^{11}$ The uranium oxide sample (named UO 18) was deposited on April 10, 2003, and was sputtered at an argon partial pressure of $2.88 \times 10^{-3}$ torr in the system named Davy. This sample was allowed to oxidize in ambient air.

The uranium nitride sample whose reflectance is reported here (named UN04) was deposited on September 30, 2003 at an argon partial pressure of $1 \times 10^{-3}$ torr and a nitrogen partial pressure of about $1 \times 10^{-5}$ torr in the second system named Joey. The residual gas composition in the system Joey after bake-out was of nitrogen, oxygen and argon at the ratios found in air as determined by a Ferran Scientific millipole analyzer (MPA). A small turbo pump was used in parallel with the Cryotorr 8 pump to remove hydrogen and helium, which are not pumped well by the cryopump. The pumping system was throttled by mostly closing the gate valve in front of the cryopump prior to sputtering. We employed a 20 -sccm full-scale mass flow controller to set the Ar pressure to the level needed to ignite the plasma. Nitrogen was flowed through a separate line to the chamber and was controlled by a low-flow, sapphire diaphragm valve immediately above the chamber. During sputtering the MPA was used to control the nitrogen flow to achieve the desired nitrogen partial pressure of $\sim 1 \times 10^{-5}$ torr. This is a pressure which has been reported as producing UN with a 1 to 1 stoichiometry while pressures a factor of ten or higher produce $\mathrm{UN}_{2}$. A quartz crystal thickness monitor in front of the shutter allowed us to achieve the desired sputter rate, usually about 0.1 to $0.4 \mathrm{~nm} / \mathrm{sec}$, by adjusting RF power to the target. More details of how the UN samples were prepared can be found in Urry. ${ }^{2}$

The target thickness for the uranium oxide sample after oxidation was about $30 \mathrm{~nm}$ as measured by $\mathrm{x}$-ray diffraction (XRD). After sputtering, the uranium film was allowed to oxidize naturally in laboratory air. Sixty- 
three days elapsed from its removal from the vacuum chamber before its thickness was measured. Prior to this report, studies of the oxidation rates of uranium thin films have been conducted. ${ }^{11}$ It should be noted that many bulk oxides of uranium are known. Even for a given composition, such as $\mathrm{UO}_{3}$, many different crystal structures are known. Also $\mathrm{UO}_{2}$ tolerates a large range of nonstoichiometry. The value of $\mathrm{x}$ in $\mathrm{UO}_{2+\mathrm{x}}$ can reach 0.25 before inducing crystal changes. Here, we denote these uranium oxide films as $\mathrm{UO}_{2}$ because the oxide compounds are closest to $\mathrm{UO}_{2}$ in stoichiometry. Therefore, when we refer to the reflectance and other properties of the uranium oxide film used in the study, it should be understood that this means, $\mathrm{UO}_{2}$. For the uranium oxide, the $\mathrm{UO}_{2}$ stoichiometry and handbook density of $10.59 \mathrm{~g} / \mathrm{cm}^{3}$ was used in calculation in accordance with Oliphant's approach. ${ }^{11}$

\subsection{Ni and Au deposition through thermal evaporation}

The nickel and gold films were prepared by evaporating $\mathrm{Ni}$ wire from a resistively heated tungsten boat (RD Mathis Co.) in a large, cryopumped, stainless steel "bell jar" coater. The base pressure of the system was 3.2 $\mathrm{x} 10^{-4} \mathrm{~Pa}\left(2.4 \times 10^{-6}\right.$ torr $)$. A quartz crystal monitor was used to measure the evaporation rate. The source was shuttered as the voltage to the tungsten boat was increased. When the evaporation rate reached about $1-2 \mathrm{~nm} / \mathrm{s}$, the shutter was opened and the substrates were coated. Fast deposition rates are known to be preferable in obtaining the highest reflectance for aluminum and many materials. This is probably due to limiting the extent to which impurities, usually oxygen, are drawn into the film from residual air and water vapor in the vacuum. After the monitor recorded about $91 \mathrm{~nm}$ of film the voltage to the source was cut, the box was vented to air and the films removed for further study. They were allowed to naturally oxidize for two days before thickness measurement using XRD and up to several weeks before measurement at the Advanced Light Source.

At the time the first films were deposited, the tooling factor of the crystal monitor for the position of the substrates was not known. After the thickness of the film on Si was determined via XRD (see below) to be about $50 \mathrm{~nm}$ the tooling factor of the crystal monitor was seen to be about 55\%. The crystal monitor was set significantly closer to the evaporation boat than the substrates, though to one side, so this tooling factor is consistent with geometry. Since the surface roughness of polycrystalline materials usually increases with increasing film thickness and surface roughness decreases reflectance, thinner Ni films were desirable. Calculations had indicated that all Ni films thicker than about $30 \mathrm{~nm}$ should have the same low-angle, soft x-ray reflectance over our range of interest. The target thickness for the nickel film was chosen to be between 30 and $60 \mathrm{~nm}$.

Before evaporating the gold sample, a small amount of chromium was evaporated onto the substrate to aid in the adhesion of the gold layer on top. Once again, calculations showed that a gold layer of $30 \mathrm{~nm}$ was desirable. The two samples deposited on silicon test wafers are referred to as NiO-on-Ni and Au deposited.

\subsection{Determining thin-film thickness through x-ray diffraction}

Thin-film interference of reflected $\mathrm{x}$ rays was used to determine the thickness of our deposited thin films. Using a Scintag ${ }^{\circledR} \mathrm{X}$-ray diffractometer (XRD), we measured the low-angle reflectance at $\mathrm{Cu}-\mathrm{K}_{\mathrm{a}}(0.154 \mathrm{~nm})$, paying particular attention to interference maxima and minima. Subsequently, we used IMD to model our layers. ${ }^{12}$ By adjusting the thickness of the modeled layer, the number of diffraction peaks in an angle interval can be adjusted to match the number of measured peaks, giving the layer thickness. Uncertainties were calculated by how much we could vary the layer thickness and still get the desired number of peaks in the change in angle. For nickel, this thickness was $49.7 \pm 0.5 \mathrm{~nm}$. For gold, this thickness was $29.5 \pm 0.5 \mathrm{~nm}$. For the uranium oxide sample this thickness was $31.8 \pm 0.5 \mathrm{~nm}$. For uranium nitride, this thickness was $38 \pm 0.5 \mathrm{~nm}$. Lunt's six samples were used to measure the optical constants of uranium oxide and varied in thickness from 5.0 to $25.0 \mathrm{~nm}$. $^{5}$ 


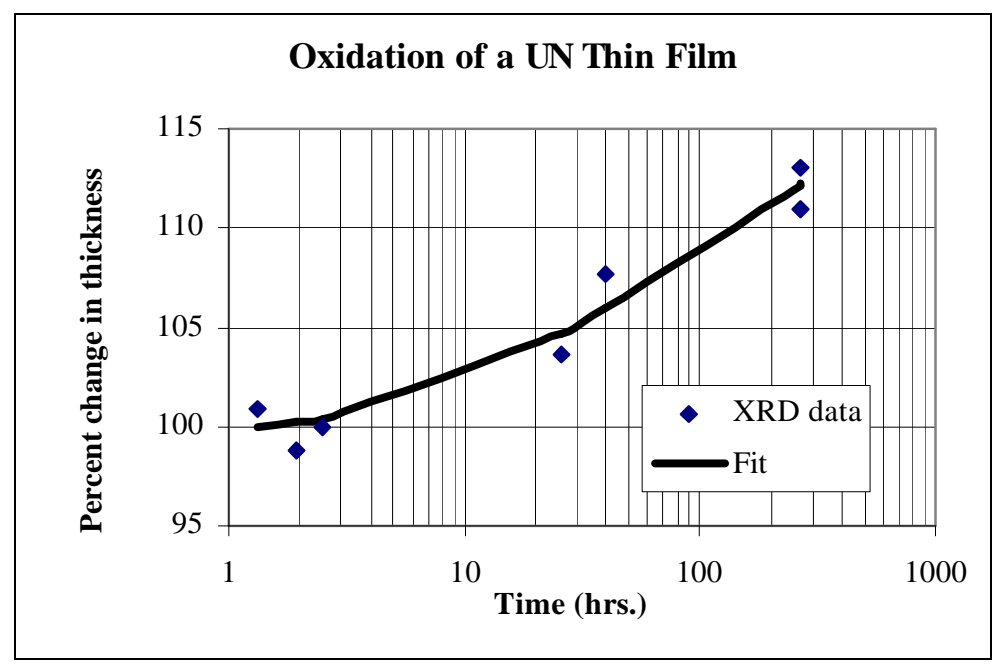

Fig. 1: Change in thickness vs. time as measured by XRD [2]

The sample UN04 was measured by XRD repeatedly over a ten-day period. The sample's thickness increased over time (see Fig. 1). We attributed this swelling to oxidation. This behavior shows that the thin-film uranium nitride sample prepared as described was not totally resistant to oxidation though it is significantly more stable than uranium metal.

\subsection{Surface roughness measurement}

Atomic force microscopy (AFM) was used to measure the surface roughness of the thin films. AFM measurements were made in tapping mode at BYU. The AFM consists of a microscopic tip on the end of a tiny cantilever that is tapped at high frequency on the surface of the samples. A laser is reflected off of the back of the tip so that as the tip goes up and down as it passes bumps and valleys in the sample, the laser light is deflected and the relative surface height can be measured. The deflection of the laser is measured using a photodiode detector. The average surface roughness (RMS) over a $100 \times 100 \mathrm{~nm}$ area of the uranium oxide sample was $0.98 \mathrm{~nm}$. For Urry's sample, the average RMS roughness was $0.40 \mathrm{~nm}$ over a $100 \times 100 \mathrm{~nm}$ area. For Lunt's uranium oxide samples, the average RMS roughness was $0.35 \mathrm{~nm}$ over a 1000 x $1000 \mathrm{~nm}$ area.

\subsection{Reflectance measurements at the Advanced Light Source}

Sample reflectance was measured at glancing angles from zero to 85 degrees (near normal) and for wavelengths between 2.1 and about $30 \mathrm{~nm}$ at the Advanced Light Source (ALS: Lawrence Berkley National Laboratory, The University of California-Berkley) on Beamline 6.3.2. The process of normalization to extract reflectances is described in more detail, along with further details on Beamline 6.3.2, at the CXRO webpage and can also be found in Underwood. ${ }^{13,14}$

A main goal of this project is to experimentally determine the index of refraction of uranium oxide and uranium nitride. The index of refraction is generally written as

$$
N=n+i \mathrm{k},
$$

where $n$ is the real part of the index of refraction and $k$, the imaginary part, is called the coefficient of absorption. When discussing the EUV, a slight variation of this is used. In the EUV $n$ is very near that of vacuum, 1, in almost all media, so it is written $n=1-\delta$ and $\delta$ is specified rather than $n$. Also $\beta$ is used instead of $k$ as the coefficient of absorption.

When $\mathrm{N}$ is known, reflectance from multiple layers can be computed using the Parratt formula and the Fresnel coefficients. ${ }^{15,16}$ The Fresnel coefficients are given for the s- and p-polarizations of light as follows: 


$$
f_{p, m}=\frac{N_{m-1}^{2} q_{m}-N_{m}^{2} q_{m-1}}{N_{m-1}^{2} q_{m}+N_{m}^{2} q_{m-1}} \quad \text { and } \quad f_{s, m}=\frac{q_{m}-q_{m-1}}{q_{m}+q_{m-1}}
$$

where $m$ is the $m$ th surface in the mirror and

$$
q_{m}=\sqrt{N_{m}^{2}-\cos ^{2} \theta_{i}} .
$$

Here $?_{i}$ is the angle from grazing of the light incident on the film. Now the recursive Parratt formula can be used:

$$
r_{s, m}=C_{m}^{4} \frac{f_{s, m}+r_{s, m-1}}{1+f_{s, m} r_{s, m-1}} \quad \text { and } \quad r_{p, m}=C_{m}^{4} \frac{f_{p, m}+r_{p, m-1}}{1+f_{p, m} r_{p, m-1}}
$$

where

$$
C_{m}=e^{i q_{m} d_{m} \pi / \lambda} .
$$

In equation (7), $d_{m}$ refers to the layer thickness and ? refers to the wavelength of incident light. Finally, reflection from the film is given using the coefficients for the $M$ th layer:

$$
R_{s}=\left|r_{s, M}\right|^{2} \quad \text { and } \quad R_{p}=\left|r_{p, M}\right|^{2} .
$$

\section{DATA}

Here we present the 2.5 to $12 \mathrm{~nm}$ portion of the reflectance data measured at ALS Beamline 6.3.2 on June 13-15, 2003, November 19-21, 2003, and February 12-15, 2004. We separate the data presented here into three figures depending upon the grazing incidence angle at $5^{\circ}, 10^{\circ}$, and $15^{\circ}$. Each chart has five lines labeled respectively as $\mathrm{UO}_{2}$ (thickness of $31.8 \mathrm{~nm}$ ), UN (thickness of $38 \mathrm{~nm}$ ), $\mathrm{NiO}$ on Ni (thickness of $49.7 \mathrm{~nm}$ ), Ir, and Au (thickness of $29.5 \mathrm{~nm})$.

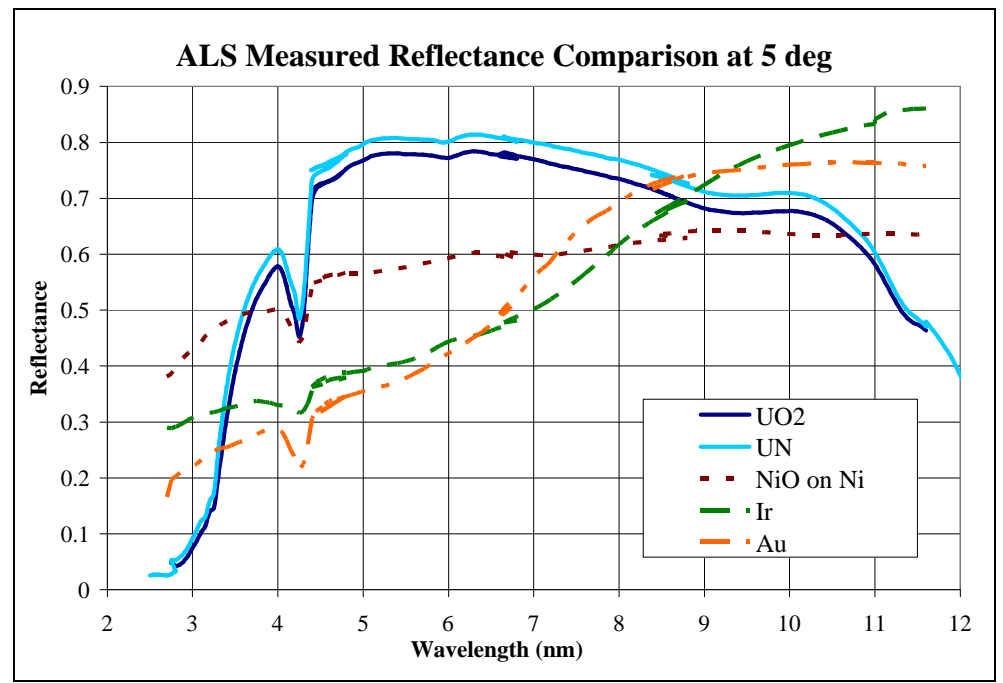

Fig. 2: Measured reflectance of $\mathrm{UO}_{2}$ from sample $\mathrm{UO18}$, UN, NiO on Ni, Ir, and $\mathrm{Au}$ at $5^{\circ}$ from 2.7 to $11.6 \mathrm{~nm}$

As shown in Fig. 2, at $5^{\circ}$ uranium oxide and uranium nitride reflect more than nickel, gold, and iridium from 3.6 to $8.5 \mathrm{~nm}$. At this lower grazing incidence angle, the uranium compounds have a maximum reflectance of about $80 \%$ from 5.2 to $6.6 \mathrm{~nm}$ as shown in Fig. 2. This value is $20-40 \%$ greater than the reflectance of nickel and even more than gold and iridium here. 
Notice the dip in reflectance of all three graphs at about $4.3 \mathrm{~nm}(285 \mathrm{eV})$. We previously suggested that this may correspond to the absorption edge of carbon near $284 \mathrm{eV}$ indicating perhaps the presence of an adventitious thin organic layer. ${ }^{3,4}$ However, XANES data, which we present below in Fig. 7, indicates that uranium might have one or more absorption resonances here. In Fig. 2, we also notice two interesting features of uranium oxide and uranium nitride. First, we notice a broad feature similar to an interference minimum at about $9.4 \mathrm{~nm}$ which we see appears more strongly at higher angles. Second, we notice a strong dip in reflection which has its minimum above $12 \mathrm{~nm}$, whereas the minimum predicted from ref. 12 is at about $11.3 \mathrm{~nm}$ with a pronounced increased by $12 \mathrm{~nm}$

The next figure (Fig. 3) shows the reflectance comparisons of uranium to the standard coatings at $10^{\circ}$ grazing incidence. First, notice once again that an absorption edge appears near $4.3 \mathrm{~nm}$. The reflectance of these uranium samples exceeds that of nickel at $5.5 \mathrm{~nm}$ and continues at nearly double the reflectance of nickel over most of this range until at $10.5 \mathrm{~nm}$ it falls below the reflectance of our $\mathrm{NiO}$ on $\mathrm{Ni}$ film. By closer examination, discrepancies in the reflectance from one wavelength range to the other can be observed. Also, we notice in Fig. 3 once again that the reflection minimum for uranium-based reflectors does not appear at $11 . \mathrm{nm}$, but above 12 nm. Furthermore, we see more pronounced the presence of a minimum in uranium's reflectance a little above 9 $\mathrm{nm}$.

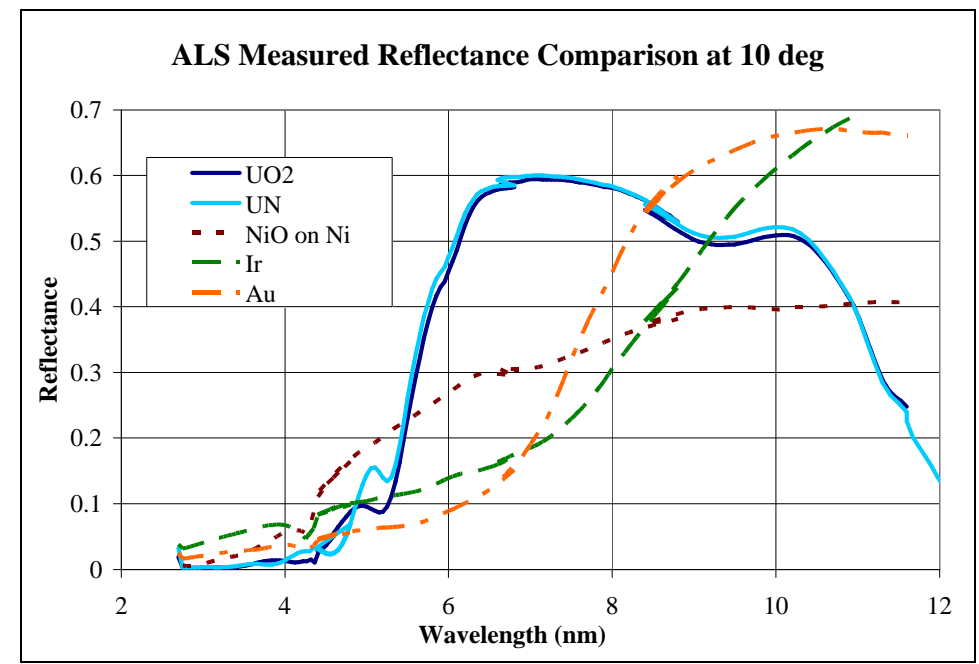

Fig. 3: Measured reflectance of $\mathrm{UO}_{2}$ from sample UO18, UN, NiO on Ni, Ir, and Au at $10^{\circ}$ from 2.7 to $11.6 \mathrm{~nm}$

At $15^{\circ}$, uranium oxide reflects more than nickel, iridium, and gold at $6.3 \mathrm{~nm}$ and more than nickel at $10.9 \mathrm{~nm}$. As seen in Fig. 4, the uranium samples reach a maximum reflectance of $33 \%$ at $10.3 \mathrm{~nm}$. The existence and significance of the reflectance minimum near $9 \mathrm{~nm}$ is discussed elsewhere. ${ }^{3,4}$

The measured reflectances of overlapping wavelength regions can be seen to differ slightly in later figures of ALS data at 4.5, 6.6, and $8.5 \mathrm{~nm}$. The bending magnet synchrotron radiation is broad band and must be monochromatized to achieve accurate reflectances. This is done by combining a grating, filter and a low-angleof-incidence triple-reflection order sorter. However, the filters and order sorters do not perfectly suppress other orders than the desired order from the grating, so the source may not be perfectly monochromatic at all wavelengths. This polychromaticity may be the cause of the slight overlap errors. Another cause of the errors could be the source hitting slightly different parts of the sample surface. 


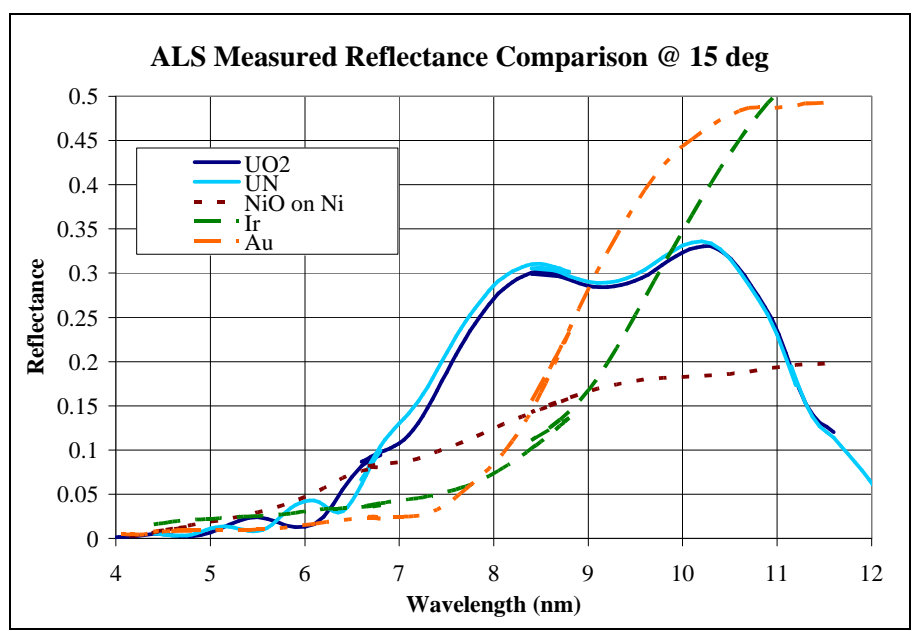

Fig. 4: Measured reflectance of $\mathrm{UO}_{2}$ from sample $\mathrm{UO18}, \mathrm{UN}$, $\mathrm{NiO}$ on $\mathrm{Ni}$, Ir, and $\mathrm{Au}$

at $15^{\circ}$ from 4.0 to $11.6 \mathrm{~nm}$

\subsection{Calculating optical constants (d and B) from reflectance angle scans}

Measurements as a function of angle (Angle scans) were also conducted on Lunt's reactively sputtered uranium oxide and on Urry's uranium nitride samples. X-ray photoelectron spectroscopy (XPS) can reveal the stoichiometry of sample surfaces. XPS examination of our UN films-not shown here but included in Urry ${ }^{2}$ and Adamson ${ }^{17}$-indicates that their surface is $\mathrm{UO}_{2}$. XPS also showed that Lunt's uranium oxide samples' surfaces had oxidized to a higher oxidation state than $\mathrm{UO}_{2}{ }^{5}$ Therefore, the structure of both of the films used in the analysis was assumed to be a bilayer structure as shown in Fig. 5. We assumed for simplicity perfectly abrupt boundaries and no interlayer roughness. Roughness was not used in this study as the few angstroms of roughness present on our samples did not affect the reflection of the samples when checked in IMD.

The measured reflectances of each of Lunt's uranium oxide sample at 9 wavelengths between $4.5 \mathrm{~nm}$ and 17.5 $\mathrm{nm}$ and at two wavelengths (13 and $14 \mathrm{~nm}$ ) for Urry's uranium nitride samples were employed in the calculation of optical constants. ${ }^{2,5}$ The thickness of the top oxide was determined by fitting its thickness, without including the change in density, using data from all six of Lunt's samples and fitting at each wavelength of interest. The thickness values obtained in this manner, from $4.4-5.5 \mathrm{~nm}$, were averaged to get $5.0 \mathrm{~nm}$. This value was fixed as the thickness of the top oxide in all subsequent fits.

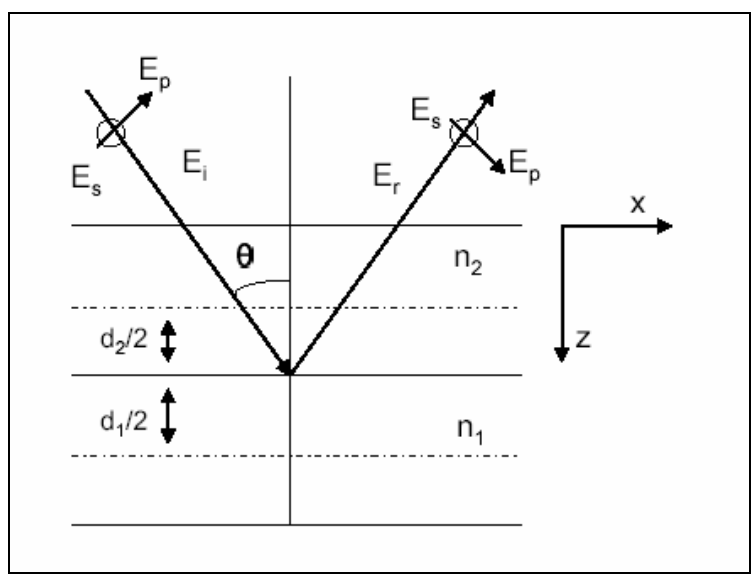

Fig. 5: Geometry for Reflection Calculations [5] 
Calculations were then performed using angle scan data from all six samples to determine the best $d$ and $\beta$ for both the UO2 layer and for the top layer of unknown oxide. These are in a MATLAB file that can be obtained in Lunt. ${ }^{5}$ The basic approach was to minimize the difference between the measured reflectance of the angle scans with calculated reflectance by adjusting the values for $d$ and $\beta$. Published constants were used as the initial values of $d$ and $\beta .{ }^{13}$ A similar process was followed to determine the constants for uranium nitride with additional information being available in Urry. ${ }^{2}$ The measured optical constants for uranium nitride and uranium oxide are shown below. The optical properties of UN and an oxidized top layer extracted from Urry's sample are shown in Tables 1 and 2 respectively. The optical properties of Lunt's oxidized top layer and the $\mathrm{UO}_{2}$ under layer are shown in Tables 3 and 4 respectively.

\begin{tabular}{|c|c|c|}
\hline $\boldsymbol{?}(\mathbf{n m})$ & $\delta$ & $\beta$ \\
\hline 13 & 0.01152 & 0.0595 \\
\hline 14 & 0.0138 & 0.0416 \\
\hline
\end{tabular}

Table 1: $d$ and $B$ of $U N$ calculated from measured reflectance taken at the ALS as reported in M. Urry[2]

\begin{tabular}{|c|c|c|}
\hline $\boldsymbol{?}(\mathbf{n m})$ & $\delta$ & $\beta$ \\
\hline 13 & -0.0228 & .0292 \\
\hline 14 & 0.0252 & .0261 \\
\hline
\end{tabular}

Table 2: $d$ and $B$ of top oxidized uranium nitride layer calculated from measured reflectance taken at the ALS as reported in M. Urry[2]

\begin{tabular}{|c|c|c|}
\hline ? (nm) & d & B \\
\hline 4.6 & 0.0065 & 0.0011 \\
\hline 5.6 & 0.0103 & 0.0016 \\
\hline 6.8 & 0.0161 & 0.0031 \\
\hline 8.5 & 0.0295 & 0.0134 \\
\hline 10 & 0.0398 & 0.0269 \\
\hline 12.5 & 0.0206 & 0.0091 \\
\hline 14 & 0.0360 & 0.0151 \\
\hline 15.5 & 0.0495 & 0.0216 \\
\hline 17.5 & 0.0639 & 0.0338 \\
\hline
\end{tabular}

Table 3: $d$ and $B$ of uranium oxide top layer calculated from measured reflectance taken at the ALS as reported in S. Lunt[5]

\begin{tabular}{|c|c|c|c|c|}
\hline & \multicolumn{2}{|c|}{ ALS Measured } & \multicolumn{2}{c|}{ CXRO Calculated } \\
\hline \hline $\mathbf{?}(\mathbf{n m})$ & $\mathbf{d}$ & $\mathbf{B}$ & $\mathbf{d}$ & $\mathbf{B}$ \\
\hline 4.6 & 0.0065 & $8.09 \mathrm{E}-04$ & 0.0116 & 0.0011 \\
\hline 5.6 & 0.0103 & 0.0012 & 0.0187 & 0.0025 \\
\hline 6.8 & 0.0173 & 0.004 & 0.0302 & 0.0065 \\
\hline 8.5 & 0.0298 & 0.0151 & 0.0491 & 0.0271 \\
\hline 10 & 0.0344 & 0.0458 & 0.0674 & 0.0693 \\
\hline 12.5 & -0.0038 & 0.0129 & 0.0057 & 0.0399 \\
\hline 14 & 0.0229 & 0.0103 & 0.0509 & 0.017 \\
\hline 15.5 & 0.0362 & 0.0158 & 0.0782 & 0.0281 \\
\hline 17.5 & 0.0547 & 0.0246 & 0.1058 & 0.0464 \\
\hline
\end{tabular}

Table 4: d and B of U02 calculated from measured reflectance taken at the ALS as reported in S. Lunt[5]

\section{DISSCUSSION OF RESULTS}

The reflectance curves for $\mathrm{UN}$ and $\mathrm{UO}_{2}$ are nearly coincident. The reflectance of $\mathrm{UN}$ is greater than $\mathrm{UO}_{2}$, as shown by the reflectance of UN computed using the bulk density $\left(14 \mathrm{~g} / \mathrm{cm}^{3}\right)$. However, it is only slightly greater than $\mathrm{UO}_{2}$; whereas, calculation demonstrates that it should be $\sim 10 \%$ larger. This could be explained by the UN not being at bulk density. It may be necessary to deposit UN at high temperatures or with substrate bias to realize bulk UN density and better stability. The melting temperature of UN is about $3000 \mathrm{~K}$ so UN as deposited at room temperature is expected to be in zone 1 of the Thornton plot and, thus, could be quite porous. 


\subsection{Comparison with reflectance from previously published data}

As stated earlier, there is a feature apparent at $4.3 \mathrm{~nm}$ in the curves in Fig. 2-4. Since similar features are present in $\mathrm{Au}$, Ir, and $\mathrm{Ni}$, as well as the uranium compounds, they likely have a common origin, which we associate with carbon contamination. However, we notice that the feature is particularly pronounced in the uranium plots. In an effort to determine whether this feature can be attributed only to the presence of carbon, we modeled different structures of uranium oxide using the Center for X-ray Optics website. Fig. 6 shows a comparison of measured uranium oxide reflectance and reflectances from different structures calculated using the CXRO website. ${ }^{13}$

Notice how the presence of carbon does not accurately match the shape of the absorption resonance at about 4.3 $\mathrm{nm}$. Additionally, the amount of carbon needed to achieve the appropriate depth of the feature, $3 \mathrm{~nm}$, is unlikely to be present. An alternative explanation is that the feature is due, in part, to the presence of carbon and, in part, to the presence of one or more absorption resonances in uranium not reported in reference 13.

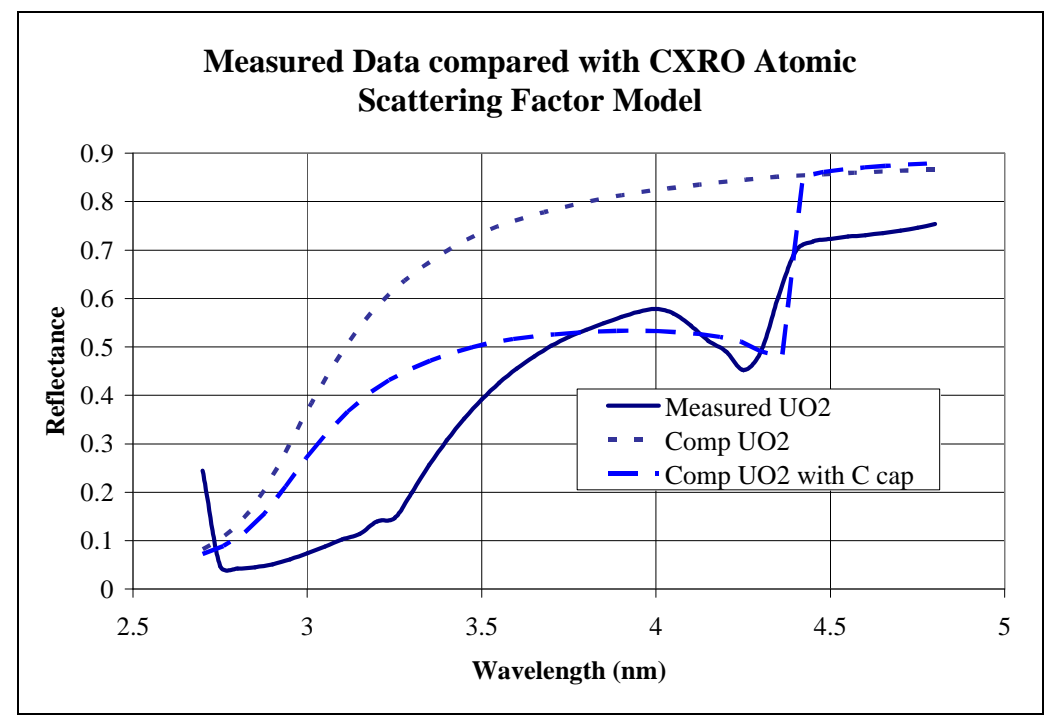

Fig. 6: Reflectance comparison of measured uranium oxide and calculated reflectance from different structures computed using the CXRO website[13]

The Handbook of Chemistry and Physics reports x-ray emissions from uranium at $294.5 \mathrm{eV}(4.21 \mathrm{~nm})$ and 286.3 $\mathrm{eV}(4.33 \mathrm{~nm}) .{ }^{18}$ Absorption features can occur near edges if the upper level is near the continuum. To ascertain whether any features in this energy range exists, a XANES (x-ray absorption near-edge structures) study of uranium oxide was performed at the ALS. XANES measures the electron current from a sample as a function of energy (or wavelength) of the incident light. As the energy is increased a point comes when photoelectrons can be emitted. Their loss from the sample is made up by current flowing from ground through a wire attached to the sample. The small current is measure in an electrometer. Fig. 7 shows the XANES data of thorium oxide and uranium oxide. The two have been normalized to carbon (we used a blank spot on the conducting carbon tape onto which the samples were attached) to minimize the effect which carbon contamination could have. As we can see, the presence of absorption features can clearly be seen in uranium near $285 \mathrm{eV}(4.3 \mathrm{~nm})$ whilst no resonance appears in thorium at this energy. We regard this as evidence that the optical constants of uranium compounds need to be investigated further in this region of the spectrum. 


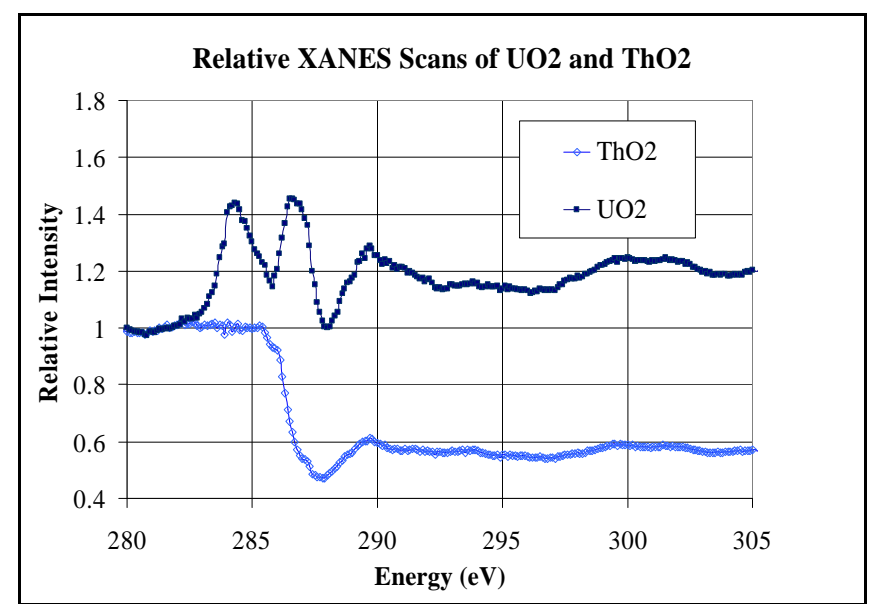

Fig. 7: X-ray Absorption at Near Edge Spectroscopy (XANES) data taken at the ALS of uranium oxide and thorium oxide

\subsection{Application for uranium based thin films}

As can be seen from the reflectance plots in Figs. 2-4, a principle application for uranium compound thin films could be for EUV and soft x-ray reflectors. As stated earlier, these reflectors should have abundant applications in astronomy, medical and biological imaging, and other glancing incidence mirrors in the 2.7-11.6 nm range.

There are, in addition, two other potential applications. Perhaps the most exciting of these two applications for uranium compounds in the EUV is in Fresnel zone plate lens. A zone plate is a microscopic plate (radii are on the order of between $100 \mathrm{~nm}$ and 100 microns depending on the photon energy of interest) consisting of concentric rings of thin material where as the radius of the ring increases the thickness of the ring decreases. There is a corresponding amount of empty space between each ring so that the ring and vacant space has the same area. These zone plates work as diffractive lenses and are crucial in many types of EUV or soft x-ray imaging systems such as EUV microscopes for biological, medical, and materials science imaging. Attwood describes in depth the theory of zone plate lenses for the EUV (see Chapter 9). ${ }^{20}$

Theory predicts that for a zone plate with perfectly absorptive rings, the efficiency of each order (i.e. the ratio of the intensity of light focused into the $\mathrm{m}^{\text {th }}$ focal point compared to the intensity of light incident upon the lens) is given by

$$
\eta_{m}=\left\{\begin{array}{cc}
0.25 & m=0 \\
\frac{1}{m^{2} \pi^{2}} & m=\text { odd } \\
0 & m=\text { even }
\end{array}\right.
$$

where $m$ is the diffractive focusing order. As we can see, the zeroth order receives $25 \%$ of the light (or in other words, $25 \%$ of the light passes straight through), the odd orders receive $1 / \mathrm{m}^{2} \mathrm{p}^{2}$ percent of the light, with about $10 \%$ focusing into the first order. $50 \%$ of the light is absorped by the zone plate. However, for zone plates made of materials that are partially transparent, this order of efficiency can greatly increase as described by Attwood ${ }^{20}$ and Kirz. ${ }^{19}$ Due to uranium's low beta, it is less absorptive in this region than many substances. Kirz shows that the diffractive order $m$ efficiency of trasmissive zone plates is given by

$$
\eta_{m}=\frac{1+\exp \left(-2 \frac{\beta \phi}{\delta}\right)-\cos (\phi) \exp \left(-\frac{\beta \phi}{\delta}\right)}{m^{2} \pi^{2}}
$$

where $m$ is the diffractive order, $d$ and $\beta$ are the materials optical constants, and $\phi$ is given by

$$
\phi=2 \pi \frac{t \delta}{\lambda}
$$


with zone plate thickness $t$ and wavelength ?. The efficiency of our uranium samples can be computed at the values for $d$ and $\beta$ given by Lunt for $\mathrm{UO}_{2}$ and for the unknown uranium oxidation state of her sample's top layer (probably a combination of $\mathrm{UO}_{2}$ and $\mathrm{UO}_{3}$ ). ${ }^{5}$ The efficiencies of $100 \mathrm{~nm}$ zone plates were computed using equations 3.2 and 3.3 and the constants from the CXRO webstite for gold, nickel, and germanium and are found in Table 5. ${ }^{13}$ The wavelengths and efficiencies are highlighted for those energies where uranium has a higher first-order efficiency than gold, nickel, and germanium.

\begin{tabular}{|c|c|c|c|c|c|}
\hline $\begin{array}{c}\text { Energy } \\
(\mathbf{e V})\end{array}$ & $\mathbf{A u}$ & $\mathbf{N i}$ & $\mathbf{G e}$ & UO $_{\mathbf{2}}$ & UO Top \\
\hline 99.2 & 0.1013 & 0.1013 & 0.1013 & 0.0971 & 0.0925 \\
\hline 124 & 0.0970 & 0.1013 & 0.1014 & 0.1013 & 0.1015 \\
\hline 145.9 & 0.1098 & 0.1013 & 0.1020 & 0.1000 & 0.0989 \\
\hline 182.4 & 0.1219 & 0.0998 & 0.1049 & 0.1781 & 0.1771 \\
\hline 221 & 0.1112 & 0.0985 & 0.1117 & 0.2857 & 0.2623 \\
\hline 270 & 0.1013 & 0.1311 & 0.1160 & 0.1811 & 0.1710 \\
\hline
\end{tabular}

Table 5: Computed first-order efficiency for $100 \mathrm{~nm}$ thick zone plates of various materials

As can be seen from Table 5, the efficiencies potentially achieveable for uranium oxide at 182.4, 221, and 270 $\mathrm{eV}$ are much higher than the efficiencies of other commonly suggested materials. At $221 \mathrm{eV}(5.6 \mathrm{~nm})$, uranium more than doubles the efficiency of the other materials.

Another potential application for uranium in the EUV is as a notch filter. A notch filter is made of a system consisting of a thin-film filter whose absorption edge matches the reflectance fall off of an associated mirror (see Attwood pages 78 and 79 for more discussion). ${ }^{20}$ These filter-mirror pairs are moderate pass filters which block the photons of energy above the mirror's reflectance fall off and below the filter's absorption edge. Uranium compounds might provide the mirror to such a notch filter at between 3-5 nm depending upon the incident angle (see Figs. 20-22) and perhaps at the other reflectance fall off at $15 \mathrm{~nm}$. Uranium compounds might provide the filter portion of a notch filter at its strong absorption edge at $12 \mathrm{~nm}$.

\section{CONCLUSIONS}

Uranium thin-film reflectors were found to be more reflective than traditional thin-film coatings (nickel, iridium, and gold) over a large range of angles and wavelengths as predicted by the published constants. Specifically, uranium oxide and uranium nitride reflect more than standard coatings at $5^{\circ}$ grazing incidence from 4.5 to 8.5 $\mathrm{nm}$, at $10^{\circ}$ from 5.5 to $9 \mathrm{~nm}$, and at $15^{\circ}$ grazing incidence from 6.5 to $9 \mathrm{~nm}$. Uranium-based coatings have been used successfully as EUV reflectors and hold great promise in further applications in astronomical, technological, or medical optics. Calculated optical constants are given for uranium oxide and uranium nitride between 4.6 and $17.5 \mathrm{~nm}$. Due to the high reflectance of uranium based thin films from 4 to $12 \mathrm{~nm}$, we recommend that uranium-based mirror coatings should be developed and implemented for future projects where broadband, low angle, soft x-ray mirrors are required. Additionally, uranium-based thin films show great promise for use in Fresnel zone plates and notch filters. Another finding of this report is that the complex indices of refraction probably differ noticeably from the reported values from the published constants. Evidence is shown that an absorption resonance exists in uranium at $4.3 \mathrm{~nm}(283 \mathrm{eV})$. Additionally, we see that the published constants do not reproduce the reflection minimum at about $9.5 \mathrm{~nm}$ in the uranium compounds reflectance measurements and that the absorption edge is not at $11 \mathrm{~nm}$, as reported by the model. We are in the process of determining the index of refraction for naturally oxidized uranium and uranium nitride over a larger portion of the soft x-ray and EUV range in conjunction with the Center for X-ray Optics. Furthermore, we conclude that thin-film uranium nitride is slightly unstable against oxidation under the preparation techniques used in atmosphere and oxidizes slowly to uranium oxide. This was seen using XRD to find a change in thickness of $12 \%$ in 10 days. XPS also showed that the surface of the UN film had become $\mathrm{UO}_{2}$. The oxidation 
rate could be further studied by using XPS depth profiling, or by making more XRD and TEM measurements as a function of air-exposure time. It would also be useful to take measurements nearer the time of the ALS runs so that thickness and composition are found directly rather than extrapolated from older measurements. ${ }^{2}$

\section{ACKNOWLEDGEMENTS}

We are grateful to Hollilyn Drury and Megan Rowberry for aiding in the sputtering the air-oxidized uranium films studied. An SPIE scholarship and a BYU ORCA Scholarship to Richard Sandberg and BYU Department of Physics and Astronomy research funds aided in the research. We also acknowledge gratefully the financial contributions of V. Dean and Alice J. Allred and Marathon Oil Company (US Steel) for gifts to Brigham Young University for thin-film research. We thank all the members of the BYU EUV team for their support, especially Kristi Adamson for XPS, Jed Johnson and Luke Bissell for sputtering films and ALS measurements, Nikki Farnsworth, Mindy Tonks for AFM, and Winston Larsen for TEM. We thank Dr. Ritva Keski-Kuha for providing the sputtered iridium samples. We are grateful to Eric Gullikson and Andy Aquila at ALS Beamline 6.3.2 at LBNL for their help in data interpretation, reduction, and analysis.

\section{REFERENCES}

${ }^{1}$ D. D. Allred et al., in A. K. Freund, A. T. Macrander, T. Ishikawa, and J. T. Wood (eds.) X-ray Mirrors, Crystals and Multilayers, Proc. SPIE, 4782 (2002) 212-223.

${ }^{2}$ M. K. Urry, "Determining Optical Constants of Uranium Nitride Thin Films in the Extreme Ultraviolet (1.6-35 nm)," (Senior Thesis) Dept. of Physics and Astronomy, Brigham Young University, 2004.

${ }^{3}$ R. L. Sandberg et al., in A. M. Khounsary, U. Dinger, K. Ota (eds.) Advances in Mirror Technology for X-Ray, EUV Lithography, Laser, and Other Applications, Proc. SPIE, 5193 (2003) 191-203.

${ }^{4}$ R. L. Sandberg, et al., in SYNCHROTRON RADIATION INSTRUMENTATION: Eighth International Conference on Synchrotron Radiation Instrumentation, San Francisco, 2003, AIP, (2004) 796-799.

${ }^{5}$ S. Lunt, "Determining the Indices of Refraction of Reactively Sputtered Uranium Dioxide Thin Films from 46 to 584 Angstroms," (Masters Thesis) Dept. of Physics and Astronomy, Brigham Young University, 2002.

${ }^{6}$ Webster Cash, University of Colorado, Personal Communication.

${ }^{7}$ E.H.P. Cordfunke, The Chemistry of Uranium, Elsevier Publishing Co, Amsterdam, 1969.

${ }^{8}$ L. Black, F. Miserque, T. Gouder, L. Havela, J. Rebizant, and F. Watsin, Journal of Alloys and Compounds, $315,(2001) 36-41$.

${ }^{9}$ K. Takemoto, et al., AIP Conf. Proc. 507 (2000) 446.

${ }^{10}$ Private Communication, Ritva Keski-Kuha, Goddard Space Center.

${ }^{11}$ D. Oliphant, "Characterization of Uranium, Uranium Oxide and Silicon Multilayer Films," (Masters Thesis) Dept. of Physics and Astronomy, Brigham Young University 2000.

${ }^{12}$ Program for EUV and X-ray reflectance calculations, courtesy of Prof. David L. Windt: windt@astro.columbia.edu. http://cletus.phys.columbia.edu/windt/idl

${ }^{13} \mathrm{http} / / / \mathrm{www}-\mathrm{cxro}$.lbl.gov/als6.3.2/, July, 2003 or contact Eric Gullikson from the webpage for additional questions.

${ }^{14}$ J.H. Underwood et al., Rev. Sci. Instrum., 67-9 (1996) 1-5.

${ }^{15}$ V.G. Kohn. "On the theory of reflectivity by an x-ray multilayer mirror," Phys. Stat. Sol. 185 (61), 61-70 (1995).

${ }^{16}$ L.G. Parratt. "Surface studies of solids by total reflection of x-rays," Physical Review 95 (2), 359-369 (1954).

${ }^{17}$ K.R. Adamson, "Determining Chemical Composition through X-Ray Photoelectron Spectroscopy," (Senior Thesis) Dept. of Physics and Astronomy, Brigham Young University, 2004.

${ }^{18}$ D. R. Lide (ed.), CRC Handbook of Chemistry and Physics, Section 10, 71st edn. CRC Press, Boca Raton, 1990-91, 256.

${ }^{19}$ J. Kirz, JOSA 64, 301 (1974).

${ }^{20}$ D. Attwood, Soft X-rays and Extreme Ultraviolet Radiation (Cambridge University Press, Cambridge, 1999). 